\title{
STUDY OF THE NORMALITY AND HOMOGENEITY OF LEVELLING ERRORS (SUMMARY)
}

\author{
A. M. Wassef and F. Z. A. Messih
}

\section{Survey of Egypt, Giza}

(1) Most statistical methods of analysis suggested for use in levelling are based on the normal law. To test the normality of the actual distribution of, say, the aggregate of the values of $w_{i j}=\rho_{i j} / R_{i j}$ the four moments $\mu_{1}, \mu_{2}, \mu_{3}$ and $\mu_{4}$ are calculated from the equations

$\mu_{1}=0, \mu_{2}=\nu_{2}-\nu_{1}{ }^{2}, \mu_{3}=\nu_{3}-3 \nu_{1}, \nu_{2}+2 \nu_{1}{ }^{3}, \mu_{4}=\nu_{4}-4 \nu_{1} \nu_{3}+6 \nu_{1}{ }^{2} \nu_{2}-3 \nu^{4}{ }_{1}$, where $\nu_{r}=\Sigma w_{i j}^{r} / \mathcal{N}, \mathcal{N}$ being the number of $w_{i j}$ 's. For large $\mathcal{N}$, the asymmetry of the distribution and its kurtosis may be assessed from the values of $\gamma_{1}=\mu_{3} / \mu_{2}{ }^{3 / 2}$ and $\gamma_{2}=\beta_{2}-3=\left(\mu_{4} / \mu_{2}^{2}\right)-3$, with standard errors (s.e.) $\sqrt{ }(6 / \mathcal{N})$ and $\sqrt{ }(24 / \mathcal{N})$ respectively. Sharply peaked long-tailed curves have $\beta_{2}>3$ and are called lepto-kurtic, comparison being made with the normal distribution for which $\beta_{2}=3$.

Analysis of the Nile Delta net gave the following: (mark the influence of outlying values!)

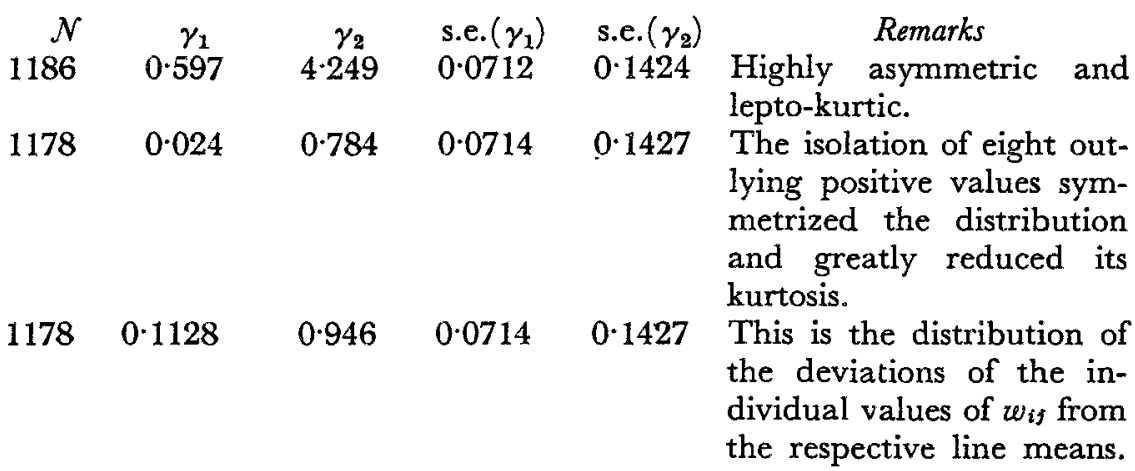

(2) To test the equality of a set of $k$ variances, the quantity

$$
M=\mathcal{N} \ln \left[\sum_{i=1}^{k} \frac{\nu_{i} S_{i}^{3}}{\mathcal{N}}\right]-\sum_{i=1}^{k}\left(\nu_{i} \ln S_{i}^{2}\right)
$$

where $\nu_{1}$ is the number of degrees of freedom on which the estimation of the variance $s_{i}^{2}$ is based, and $\mathcal{N}=\sum_{i=1}^{k} \nu_{\nu_{i}} M$ is distributed like $\chi^{2}$ with $k-1$ degrees of freedom.

The value of $M$ in the Nile Delta net was 257 with 85 degrees of freedom; but when the eight outlying values $w_{i j}$ were isolated $M$ reduced to 186 which remains significant $(M=131.0$ at $0.1 \%$ level $)$.

Weighting $w_{i j}$ in proportion to $R_{i j}$ eliminated this heterogeneity.

The full text of this paper will be published. 